\title{
BMJ open Implementing surgical services in a rural, resource-limited setting: a study protocol
}

\author{
Duncan Smith-Rohrberg Maru, ${ }^{1,2}$ Ryan Schwarz, ${ }^{1,3}$ Dan Schwarz, ${ }^{1,4}$ \\ Jason Andrews, ${ }^{1,5}$ Maria Theresa Panizales, ${ }^{6}$ Gregory Karelas, ${ }^{1}$ \\ Jesse Stark Brady, ${ }^{1,7}$ Selwyn Rogers ${ }^{6}$
}

To cite: Maru DS-R,

Schwarz R, Schwarz D, et al. Implementing surgical services in a rural, resource-limited setting: a study protocol. BMJ Open 2011:1:e000166. doi:10.1136/bmjopen-2011000166

- Prepublication history for this paper is available online. To view these files please visit the journal online (http:// bmjopen.bmj.com).

Received 11 May 2011 Accepted 5 July 2011

This final article is available for use under the terms of the Creative Commons Attribution Non-Commercial 2.0 Licence; see http://bmjopen.bmj.com

For numbered affiliations see end of article.

Correspondence to Jesse Stark Brady; jesse@nyayahealth.org

\section{ABSTRACT}

Introduction: There are well-established protocols and procedures for the majority of common surgical diseases, yet surgical services remain largely inaccessible for much of the world's rural poor. Data on the process and outcome of surgical care expansion, however, are very limited, and the roll-out process of rural surgical implementation in particular has never been studied. Here, we propose the first implementation research study to assess the surgical scale-up process in the rural district of Achham, Nepal. Methods and analysis: Based primarily on the protocols of the WHO's Integrated Management for Emergency and Essential Surgical Care (IMEESC), this study's threefold implementation strategy will include: (1) the core IMEESC surgical care program, (2) community-based follow-up via health workers, and (3) hospital-based quality improvement programs. The implementation program will employ additional emergency and surgical care protocols developed collaboratively by physicians, nurses and the authors. This strategy will be referred to as IMEESC-Plus. This study will employ both qualitative and quantitative research methodologies to collect clinical data and information on the reception and utilisation of services. The first 18 months of the implementation process will be studied and divided into an initial phase (first 6 months) and a consolidation phase (subsequent 12 months).

Discussion: This study aims to describe the logistics of the implementation process of IMEESC-Plus, and assess the quality of the resulting IMEESC-Plus services during the course of the implementation process. Using data generated from this study, larger, multi-site implementation studies can be planned that assess the scale-up of surgical services worldwide in resource-limited areas.

\section{BACKGROUND}

Worldwide, $11 \%$ of death and disability are attributable to surgically curable diseases. Every year, 234 million major surgeries are performed, ${ }^{1-3}$ yet the distribution of these surgeries is highly inequitable. Approximately

\section{ARTICLE SUMMARY}

\section{Article focus}

- Analysis of program results utilising the WHO's Integrated Management of Emergency and Essential Surgical Care (IMEESC) approach, which will be supplemented with additional community-based follow-up protocols, and hospital-based quality improvement mechanisms; this approach will be referred to as IMEESC-Plus.

- Study of the logistics of the implementation process of IMEESC-Plus.

- Assessment of the quality of the resulting IMEESC-Plus services during the course of the implementation process.

Key messages

- There is an acute need to study the basic operational processes by which surgical services deployment occurs in remote, resource-limited settings.

- The proposed study protocol will be the first to directly address this need.

- Using data generated from this study, larger, multi-site implementation studies can be planned that assess the scale-up of surgical services worldwide in resource-limited areas.

Strengths and limitations of this study

- As the setting of this prospective study faces many of the challenges found globally in geographically-isolated, resource-poor settings, the findings may be generalisable to other sites throughout the world.

- By utilising both quantitative data and qualitative study techniques, this protocol will enable a broad analysis of program effectiveness.

$30 \%$ of the world's population receives $74 \%$ of the world's surgical procedures, with the poorest third obtaining a meagre $3 \% .^{2}$ Two billion people worldwide, roughly a third of the global population, live in areas with less than one operating room per 100000 people. As a result, patients in poor countries do not 
receive timely surgical services and suffer significant morbidity and mortality for preventable and treatable conditions. ${ }^{45}$

A massive global scale-up of surgical capacity is required to close this gap in surgical accessibility. ${ }^{6-8}$ Due to challenges regarding human resources, transportation systems and the procurement of electricity and water, implementing surgical services in resource-limited environments presents unique challenges. Although there is a growing literature on the deployment of surgical quality initiatives within existing surgical programs, data examining how to achieve this scale-up are limited. ${ }^{9}$ There is thus an acute need to study the basic operational processes by which surgical services deployment occurs in remote, resource-limited settings.

This study will be the first to describe and assess the implementation of surgical services in a resource-limited practice environment. We believe there are two components missing from the WHO's existing Integrated Management of Emergency and Essential Surgical Care (IMEESC) program: explicit mechanisms for community-based follow-up and explicit mechanisms for quality improvement. In our experiences, real-world implementation requires greater attention to these aspects of surgical care. Hence, this study will implement an IMEESC-Plus protocol at a district-level hospital in remote, rural Nepal.

To examine this scientifically, we will use mixed qualitative and quantitative implementation research methodologies. Implementation research is the study of processes for integrating empirically supported treatments into usual care, and holds high promise for reducing the gap between technological discoveries and clinical practice. ${ }^{10}$ Such research will form the scientific basis for future multi-site studies that can serve as a foundation for global financing and roll-out of surgical services in resource-limited areas worldwide.

\section{METHODS AND ANALYSIS \\ Site}

The surgical scale-up will take place in the district of Achham, located in the mountains of western Nepal, which has suffered the consequences of war, longstanding poverty and a concentrated HIV epidemic. Citizens of this region continue to struggle to gain access to basic health services, and the recently opened Bayalpata Hospital is one of the few healthcare institutions in the district of 266000 people. This facility serves as the major referral centre for Achham and its surrounding two districts. The hospital operates as a private-public partnership between the Ministry of Health of Nepal and the Nepali non-profit organisation Nyaya Health Nepal. The hospital grounds are owned by the government of Nepal. The hospital is financed by the government of Nepal (approximately 25\%), individual donors via the US-based parent organisation Nyaya Health International (approximately 50\%) and foundation grants (approximately 25\%).
Services are provided to patients of Bayalpata Hospital free of charge. These services include outpatient and inpatient services, $24 \mathrm{~h}$ emergency and delivery services, ${ }^{11}$ laboratory diagnostics, ${ }^{12}{ }^{13}$ x-ray and ultrasound services, an on-site pharmacy, ambulance service and community health programs. ${ }^{5}$ Minor surgical procedures are performed, including the repair of lacerations, drainage of abscesses, closed reductions, casting and manual vacuum aspiration. Staffing includes two physicians, two mid-level providers, six nursemidwives and approximately 20 additional staff members. ${ }^{5}$ Currently, there is no operating room, surgeon, nurse anaesthetist, or capacity for major surgeries at Bayalpata Hospital. Owing to poor roads, lack of motorised transportation, a dearth of other local healthcare facilities and widely dispersed villages, more than $20 \%$ of Bayalpata Hospital's patients walk 10 or more hours each way to receive services. The hospital oversees a network of approximately 50 community health workers (CHWs) who are part of the government's Female Community Health Volunteer program. The setting of this study presents many of the challenges faced globally in geographically isolated, resource-poor settings. Findings of the prospective study may, therefore, be generalisable to other sites throughout the world.

Implementation plan: IMEESC-PIus

\begin{tabular}{|c|}
\hline IMEESC-Plus \\
IMEESC core surgical care program \\
PLUS Community-based follow-up via health workers \\
PLUS Hospital-based quality improvement programs \\
\hline
\end{tabular}

The integrated threefold approach of Bayalpata Hospital's surgical implementation program is based primarily upon the WHO's IMEESC program. ${ }^{14}$ This strategy will be supplemented with additional community-based follow-up protocols utilising an existing CHW network and hospital-based quality improvement mechanisms. In this way, it will be possible to provide outreach services to improve patient outcomes and to establish an ongoing mechanism for institutional change. Such outreach services are critical to continuity of care in rural or remote areas with poor transportation infrastructure. The primary clinical manual utilised throughout this process will be the WHO's Surgical Care at the District Hospital ${ }^{15}$ guidelines. Protocols for operations and management policies will include the WHO's Best Practice Protocols: Clinical Safety Procedures ${ }^{16}$ as well as emergency and surgical care protocols for physicians and nurses developed in collaboration with the authors. Utilising these foundations, the Bayalpata Hospital is implementing an IMEESC-Plus program that includes: (1) the core IMEESC surgical care program; (2) community-based follow-up via CHWs; and (3) hospital-based quality improvement programs. 
Bayalpata Hospital currently has the capacity to conduct only minor surgical procedures, and the expanded services to be made available will include the procedures described in box 1 . All of these procedures will be performed by a generalist physician with postgraduate surgical training (referred to as an 'MD-GP' in Nepal). The government of Nepal is focussing on this type of practitioner to provide essential surgical care in rural areas. Surgical aspects of MD-GP training focus specifically on the surgical procedures outlined in box 1 , and competency in these procedures is required for graduation. The level of training analogous to that of MD-GP practitioners is also the focus of the IMEESC program worldwide. ${ }^{5}$ The surgeon will receive additional on-site support and training from visiting senior surgeons from among Nyaya Health colleagues in the United States and Nepal. Utilising Nyaya Health's existing Mortality and Morbidity program, ${ }^{11}$ email discussion and support will also be available to the surgical staff on a non-urgent basis. This program is intended to enable enhanced learning and reflection rather than to provide active, real-time management.

The MD-GP will oversee a nurse anaesthetist trained within the government's scheme to deliver basic anaesthesia care to surgical patients in rural areas. A staff nurse (a specific designation within the government of Nepal's healthcare provider hierarchy) will assist the MD-GP and nurse anaesthetist in providing post-operative care. In keeping with current policy, any cases that are outside the scope of practice of this modest team will be sent via ambulance to an appropriate referral centre (between 6 and $14 \mathrm{~h}$ away).

As Bayalpata Hospital has done before, the opening of the operating room will be advertised over the local radio. In our experience, this method has worked well at spreading health messages in Achham. Over time, as experience builds, Bayalpata Hospital will additionally train its CHWs as well as providers within the government's clinic network in screening for and identifying potential elective surgical candidates.

The existing network of CHWs at Bayalpata Hospital will follow-up patients after surgery to detect and prevent complications. ${ }^{5}$ Within each political subdivision, these health workers report to community health advocates,

Box 1 List of essential surgical services during scale-up at Bayalpata Hospital

Amputation of distal or proximal limbs

Appendectomy

Caesarean section

- Cholecystectomy

Exploratory laparotomy

- Hernia repair

- Hydrocele reduction

- Surgical correction of head, chest and abdominal trauma

Surgical management of acute closed and open fractures

Surgical management of wounds and burns who in turn report to hospital-based coordinators. This network was built based on the government of Nepal's existing health worker infrastructure. As such, this model should be scalable both within Nepal and in the many countries that have government-sponsored CHW networks.

The implementation of surgical services will occur within the framework of quality management using PlanDo-Study-Act (PDSA) and Root Cause Analysis (RCA) models. ${ }^{12} 13 \quad 17$ Methods will include regular data management reviews, clinical- and operational-level checklists, and a mortality and morbidity conference program. We anticipate that these will evolve over the course of the study, as with any quality improvement initiative. Bayalpata Hospital maintains an active database of administrative and clinical data that integrates outcomes for its clinical programs. In addition, quality checklists will serve as the basis for ongoing improvement, and will cover materials management, facilities maintenance, biomedical engineering and provider-level clinical care. ${ }^{18}$ Finally, an ongoing mortality and morbidity review program conducted at the hospital will serve as an institutional quality improvement mechanism. The overall program aims to identify and address challenges and action items regarding structure, process and outcomes. ${ }^{19} 20$

\section{Study participants}

The participants of this research will be patients and their families, as well as hospital staff. The main benefit to research participants will be an improvement in the accessibility, quality and safety of surgical care delivery in their communities.

All surgical patients who present to Bayalpata Hospital during the study period will be included as participants. The anticipated surgical patients will be predominantly women and children, who represent $75 \%$ of all patients served at Bayalpata Hospital. The reason for this demographic predominance is because most of the region's men travel to India as migrant workers and are thus absent from the district for large periods of time. Although paediatric patients represent a large portion of the patients seen at Bayalpata Hospital, ${ }^{21}$ children are less prone to conditions amenable to surgery and will thus constitute fewer surgical patients. Their involvement in the study, however, will be proportional to the burden of surgical disease among children in Achham.

Patients at this facility are all ethnic Nepalis due to the geographically isolated nature of the hospital. Hence, this study will be based on the demographics of the region's residents.

\section{Objectives}

This study will follow the first 18 months of the implementation process. An implementation research approach $^{10}$ will be used to study the deployment of surgical services in this rural, resource-limited setting. Since effective implementation hinges on quantifiable 
systems and human implementation as well as the reception of such systems, a mixed qualitative and quantitative research methodology will be employed. Both approaches are crucial and will be used in a complementary fashion to collect data and identify key drivers for implementation elsewhere. Given that some changes are anticipated during the initial scale-up process, the analysis will be broken down into an initial phase (first 6 months) and a consolidation phase (subsequent 12 months).

The primary aims of the study are twofold. The first goal is to describe the logistics of the implementation process of IMEESC-Plus. Logistical topics to be examined include the conditions with which patients present as well as the financial, staffing, pharmaceutical and consumable supply inputs required to address these conditions. $^{22}$ Such data have yet to be described and will be critical in assessing the resources required to plan a larger study and bring IMEESC-Plus to scale. The study's second aim is to assess the quality of the resulting IMEESC-Plus services during the course of the implementation process. The quality measures used will be the following: adherence of staff to resuscitation and surgical operating protocols, performance of morbidity and mortality conferences, patient follow-up rates, rates of complications and supply chain reliability. A mixed qualitative and quantitative approach will be employed to achieve these two aims. Using data generated from this study, larger, multi-site implementation studies can be planned to assess the scale-up of surgical services in resource-limited areas worldwide.

\section{Specific aim 1}

Specific aim 1 is to describe the logistics of the implementation process of IMEESC-Plus: the conditions with which patients present and the financial, staffing, pharmaceutical and consumable supply inputs required to address these conditions.

To achieve this aim, we will describe the types of surgical diseases, determine raw financial inputs, assess consumable and pharmaceutical needs and analyse the deployment process from the staff perspective.

\section{Nature of surgical disease presenting at Bayalpata Hospital}

This study will quantify the type of surgical diseases present and the treatment of these diseases using a simple data recording instrument (see table 1 ). This recording instrument is currently being implemented at Bayalpata Hospital for existing minor surgical services. Data will be displayed in two forms: (1) a tabular form with demographic and descriptive information, and (2) a summary form, in which information will be categorised by types of conditions present during the initial and consolidation phases. We hypothesise that there will be a gradual expansion over time of more complex diagnoses and surgical procedures. We expect that this expansion will occur rapidly during the first 6 months and hit a plateau by 18 months. This hypothesis predicts that by the end of the studied 18 months, the annual number of surgeries will approach 20 per 10000 citizens.

For this specific study, we will not be conducting a community-wide surveillance of surgical disease burden for either planning or detection purposes. There is a clear need for emergency surgical services due to the lack of such services in the region. During the last 3 years, this need for surgical capacity has become extremely clear to Nyaya Health providers. Given that Bayalpata Hospital serves a dispersed catchment area of up to 500000 people in a travel radius of over $24 \mathrm{~h}$, a community survey may not accurately predict surgical volume. Furthermore, we believe that understanding the burden of surgical disease presenting for medical services nevertheless provides important data that may aid health ministries, hospitals and non-governmental organisations seeking to roll out surgical services in resource-limited settings. Basic numbers will be obtained from mathematical models and existing healthcare utilisation data.

Most patients eligible for elective surgery (for conditions which could potentially be detected by a community survey) will ultimately be recruited through the standard government mechanism of surgical camp advertisements.

\section{Financial inputs}

All raw financial inputs into the system will be quantified and broken down into categories for pharmaceuticals, capital equipment, consumables, and facilities construction and maintenance inputs. These inputs will be obtained by monthly analyses of the financial databases currently in existence at Bayalpata Hospital. Costs will include pharmaceutical facility staffing, ${ }^{23}$ consumable materials and capital equipment; total monthly costs will be displayed in a histogram. Within each bar in the histogram, the percentage of the total costs for each category of expenditures will also be displayed. Based on Bayalpata Hospital's costing model (available for down$\operatorname{load}^{2425}$ ), we hypothesise that the overall construction and 2-year operating costs of implementing the IMEESCPlus model will be US $\$ 0.50$ per capita in the district.

\section{Pharmaceutical and consumable item utilisation}

The usage of pharmaceutical and consumable items during the roll-out process will be tabulated based upon monthly analyses of the pharmaceutical and supply databases currently in existence at Bayalpata Hospital. Assessment will be based primarily on high-volume items, including antibiotics, suture material, scalpels and needles. Monthly utilisation patterns will be displayed by means of a histogram.

\section{Qualitative documentation of the implementation process}

The deployment process will be documented qualitatively, with the primary domains of analysis being the following: human resources management, supply chains, in-hospital work flows and patient-level interactions. Hospital-level analyses will be performed according to three modalities: (1) open-ended, semi-structured 
Table 1 Surgical care registry

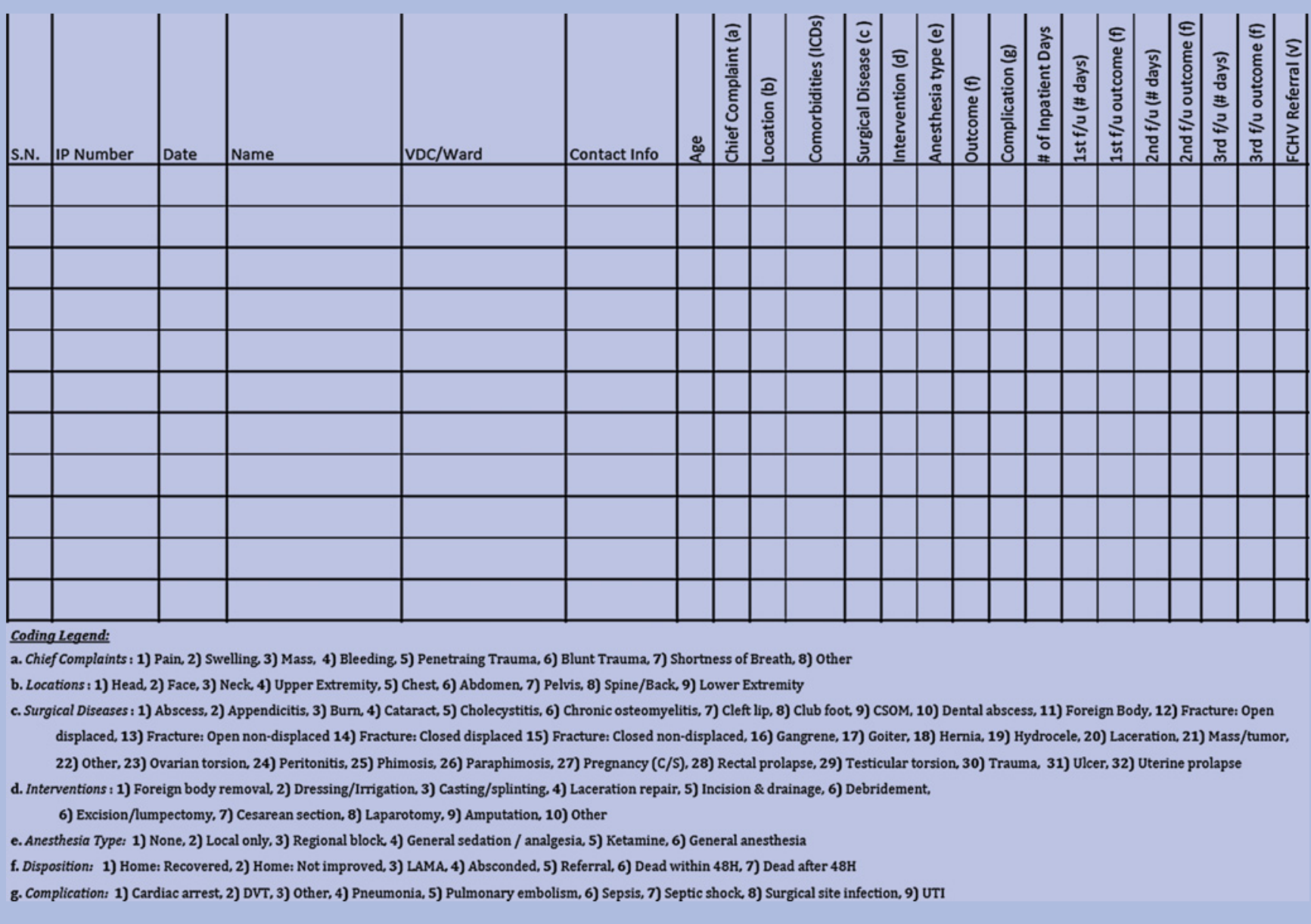

interviews of staff at 3-month intervals, (2) non-participant observation of planning meetings and morbidity and mortality conferences, and (3) focus groups with staff at 3-month intervals. ${ }^{26}$ All interviews and observations will be recorded in a journal maintained by the research coordinator. This journal will then be translated and back-translated, and assessed using the framework analysis methodology. ${ }^{22}$

\section{Specific aim 2}

Specific aim 2 is to assess the quality of the resulting IMEESC-Plus services during the course of the implementation process: adherence of staff to resuscitation and operating protocols, supply chain reliability, performance of morbidity and mortality conferences, patient follow-up rates and rates of complications.

To achieve this aim, assessments will be made of compliance with stocking protocols, adherence to safety checklists and protocols, patient-level follow-up and complication rates.

\section{Stocking protocols}

Staff compliance with stocking protocols will be assessed by adapting existing WHO site analysis tools and will be displayed via monthly histograms. It is hypothesised that there will be steady compliance with pharmaceutical and consumable stocking protocols, with no more than $5 \%$ of items missing on a monthly basis.

\section{Surgical safety and resuscitation protocols}

Assessments will be made of adherence to the Surgical Safety Checklist, as well as novel emergency resuscitation (pre- and post-surgical care) and hospital operations protocols developed for this project. The surgical safety checklist to be employed is well-validated and described, and has been adopted by the WHO as the standard for surgical services globally. ${ }^{1} 27$ However, the real-world functioning of this checklist at the outset of services in severely resource-limited settings has yet to be described. The analysis of surgical checklist adherence will occur as described in box 2. Staff adherence with other care protocols will be determined by evaluation forms that have been adapted from the WHO model to evaluate nursing and physician performance.

The rates of adherence to each protocol are estimated for the initial 6 months and the subsequent 12 consolidation months. With 100 patients in the initial period, it will be possible to estimate the true complication rate for 
Box 2 Process measures for surgical safety

Appropriate intravenous catheters

- Appropriate prophylactic antibiotics

- Peri-anaesthesia use of pulse oximetry

- Pre-anaesthesia evaluation of airway

- Post-surgical sponge count

a given process measure within (at most) a $\pm 10 \%$ interval with $95 \%$ certainty. For example, for a given process measure, if the process measure is followed in 80 out of $100(80 \%)$ patients in the initial period, then the $95 \%$ exact binomial CI will be approximately $(71 \%$ to $87 \%$ ), with a width of $16 \%$. With 400 patients in the consolidation period, it will be possible to estimate the true complication rate for a given process measure within a $\pm 5 \%$ interval with $95 \%$ certainty. For example, for a given process measure, if the process measure is followed in 380 out of $400(95 \%)$ patients, then the $95 \%$ exact binomial CI will be approximately (92\% to $97 \%$ ), with a width of $5 \%$. In this pilot phase, the adherence rates during the initial and consolidation phases will not be directly compared. Secondary logistic regression analyses will be performed to determine important predictors of complication, including acuity at admission (elective, emergency), surgery type (orthopaedic, plastic, obstetric, intra-abdominal), length and time of operation, wound classification and American Society of Anesthesiologists risk index classification.

\section{Community-based follow-up}

The rate of patient improvement will be assessed by CHW patient follow-up 1 week and 3 weeks following discharge from the hospital using formatted checklist documents and consistent data collection techniques. CHWs will be provided with structured training and hospital staff support, as currently occurs within the Bayalpata CHW program. ${ }^{28}$

Follow-up in rural Nepal is complicated by the severe lack of effective transportation infrastructure. Furthermore, given the dearth of services in the area, it is expected that patients will be travelling long distances to reach the hospital. In the first 3 months of implementation of x-ray services at Bayalpata Hospital, for example, $20 \%$ of patients travelled, typically by foot, for more than $10 \mathrm{~h}$ each way to access services. ${ }^{29}$ Despite these challenges, based on existing experience at the hospital, we hypothesise that this program will be able to provide follow-up services to $95 \%$ of patients throughout the study period.

\section{Complication rates}

Major complication rates form the basis of any surgical services monitoring and evaluation program, yet the rate at which they occur in resource-limited settings has yet to be studied. Immediate post-surgical clinical complications will be tracked as shown in box $3 .^{30} 31$ These data will be interpreted in light of the qualitative analysis of
Box 3 Immediate post-surgical outcomes measures

Acute renal failure

- Cardiac arrest requiring cardiopulmonary resuscitation

- Cerebral vascular accident

- Coma of $24 \mathrm{~h}$ duration or more

- Death

- Deep venous thrombosis

- Hospital-acquired pneumonia

- Myocardial infarction

- Pulmonary embolism

- Sepsis

- Shock

- Surgical site infection

- Systemic inflammatory response syndrome

- Transfusion of four or more units of red cells within the first $72 \mathrm{~h}$

- Unplanned intubation

- Unplanned return to the operating room

- Ventilator use for $48 \mathrm{~h}$ or more

- Wound disruption

Bayalpata Hospital's existing mortality and morbidity review program and quantitative data analysis of trends and frequency of post-operative occurrences.

Our primary outcome for this analysis will be the rate of major complications (including death, as per box 3) within the first 7 days following surgery. Patients who are lost to follow-up will be considered treatment failures, both programmatically within Bayalpata Hospital and from a research/evaluation standpoint within the context of the study. Patients and their families may, and in our experiences often do, leave earlier than the treatment team may suggest. However, even in these instances, we should be able to track down patients in their home communities. Both programmatically and scientifically, we feel that it is important to document longer than usual surgical care follow-up. A secondary outcome will be the rate of major complications prior to leaving the hospital.

With 100 patients in the initial period, it will be possible to estimate the true complication rate within a $\pm 10 \%$ interval with $95 \%$ certainty. For example, if 5 out of $100(5 \%)$ patients have a complication in the initial period, then the $95 \%$ exact binomial CI will be approximately $(1.6 \%$ to $11.3 \%$ ), with a width of $9.7 \%$. With 400 patients in the consolidation period, it will be possible to estimate the true complication rate within a $\pm 5 \%$ interval with $95 \%$ certainty. For example, if 20 out of $400(5 \%)$ patients have a complication, then the $95 \%$ exact binomial CI will be approximately $(3.1 \%$ to $7.6 \%$ ), with a width of $4.5 \%$. In this pilot phase, the complication rates during the initial and consolidation phases will not be directly compared. The complication rates are expected to be too small to fit a multiple logistic regression model to determine important predictors. However, with 20 complications in the consolidation phase, it would be possible to fit logistic regression models with each covariate separately. 


\section{Ethics and dissemination}

In this assessment of a surgical services intervention, the primary risk to subjects is the leaking of protected health information. The surgical services that patients receive are part of the standard of care practices at Bayalpata Hospital and are not research-related. Ensuring the privacy and anonymity of patient information is essential, and measures will be employed in accordance with Health Insurance Portability and Accountability $\mathrm{Act}^{32}$ (HIPAA) guidelines to ensure patient confidentiality. Such measures will include careful de-identification of patient records, use of secure servers, password-protected databases and physical locking of medical record storage in the clinic. No protected health information will be recorded or transmitted electronically. Bayalpata Hospital staff members have extensive experience in ensuring the safety of protected health information through an ongoing data management program.

An additional risk is that of psychological harm to patients who are interviewed following surgery. This will be minimised by having sensitivity training for all staff members and by using standardised instruments. Staff debriefing will also be conducted to identify challenges and encourage open discussion for support and resolution.

Ethics review board proposals will be submitted to the Brigham and Women's Hospital and to the Nepal Health Research Council prior to starting the study. All deidentified data, protocols and documentation from the study will be made openly accessible to the public and disseminated via a public-access website. ${ }^{33}$

\section{DISCUSSION}

This prospective study will be the first to describe and assess the implementation of surgical services in a resource-limited practice environment. It is proposed that there are two components missing from the WHO's existing IMEESC program: (1) explicit mechanisms for community-based follow-up and (2) quality improvement initiatives. While these components are involved to some extent, effective real-world implementation requires greater attention to these topics. Hence, the proposed study will follow an IMEESC-Plus protocol in rural Nepal that will incorporate these two additional elements.

The primary limitation of this research is that it is a single-facility study. Biases can be seen when studying the effects of a single hospital or surgeon. It is anticipated, however, that the in-depth analysis of the process and logistics of surgical scale-up implementation will form the necessary groundwork for a larger multi-site study. Furthermore, the implementation of surgical services requires a broad-based team approach, and this study will examine the entire trauma and surgical system. This system, while dependent upon individual providers, must contain set protocols that operate effectively despite the strengths and weaknesses of any given clinician. The longitudinal data on implementation process costs, safety and utilities that this study will provide are not yet available. Once determined, however, these data will provide basic parameters for designing a larger implementation research study.

Author affiliations:

${ }^{1}$ Nyaya Health, Bayalpata Hospital, Ridikot VDC, Achham, Nepal ${ }^{2}$ Department of Medicine, Brigham and Women's Hospital, Boston, Massachusetts, USA

${ }^{3}$ School of Medicine, Yale University, New Haven, Connecticut, USA ${ }^{4}$ School of Medicine, Brown University, Providence, Rhode Island, USA ${ }^{5}$ Division of Infectious Diseases, Massachusetts General Hospital, Boston, Massachusetts, USA

${ }^{6}$ Department of Surgery and the Center for Surgery and Public Health, Brigham and Women's Hospital, Boston, Massachusetts, USA

${ }^{7}$ Division of Health Sciences, Montana State University, Bozeman, Montana, USA

Funding Duncan Maru is supported by the Global Health Equity fellowship program within the Department of Medicine at Brigham and Women's Hospital.

Competing interests None.

Ethics approval An IRB proposal has been submitted to Brigham and Women's Hospital.

Contributors DM, RS, DS, JA, MP and SR worked together to conceive the study and develop the methodology. JB and GK helped to refine the methods and draft the manuscript. All authors read and approved the final manuscript.

Provenance and peer review Not commissioned; externally peer reviewed.

\section{REFERENCES}

1. Haynes AB, Weiser TG, Berry WR, et al. A surgical safety checklist to reduce morbidity and mortality in a global population. $N$ Engl $\mathrm{J}$ Med 2009;360:491-9.

2. Funk LM, Weiser TG, Berry WR, et al. Global operating theatre distribution and pulse oximetry supply: an estimation from reported data. Lancet 2010;376:1055-61.

3. Weiser TG, Regenbogen SE, Thompson KD, et al. An estimation of the global volume of surgery: a modelling strategy based on available data. Lancet 2008;372:139-44.

4. Vos T. Improving the quantitative basis of the surgical burden in lowincome countries. PLoS Med 2009;6:e1000149.

5. Chu K, Rosseel P, Gielis P, et al. Surgical task shifting in SubSaharan Africa. PLoS Med 2009;6:e1000078.

6. Galukande M, von Schreeb J, Wladis A, et al. Essential surgery at the district hospital: a retrospective descriptive analysis in three African countries. PLoS Med 2010;7:e1000243.

7. Taira B, Kelly McQueen K, Burkle F. Burden of surgical disease: does the literature reflect the scope of the international crisis? World J Surg 2009;33:893.

8. Ozgediz D, Hsia R, Weiser T, et al. Population health metrics for surgery: effective coverage of surgical services in low-income and middle-income countries. World J Surg 2009;33:1-5.

9. Luboga S, Macfarlane SB, von Schreeb J, et al. Increasing access to surgical services in sub-saharan Africa: priorities for national and international agencies recommended by the Bellagio Essential Surgery Group. PLoS Med 2009;6:e1000200.

10. Eccles MP, Armstrong D, Baker R, et al. An implementation research agenda. Implement Sci 2009;4:18.

11. Nyaya Health. Nyaya Health's Mortality Data Page. http://wiki nyayahealth.org/MortalityData (accessed 2011).

12. Kotagal $M$, Lee $P$, Habiyakare $C$, et al. Improving quality in resource poor settings: observational study from rural Rwanda. BMJ 2009;339: b3488.

13. Barker P, McCannon C, Mehta N, et al. Strategies for the scale-up of antiretroviral therapy in South Africa through health system optimization. J Infect Dis 2007;196(Suppl 3):S457-63.

14. World Health Organization. Integrated Management for Emergency and Essential Surgical Care Tool Kit. http://www.who.int/surgery/ publications/imeesc/en/index.html (accessed 2011).

15. World Health Organization. Surgical Care at the District Hospital-The WHO Manual. http://www.who.int/surgery/publications/scdh_manual/ en/index.html (accessed 2011). 
16. World Health Organization. Best Practice Protocols: Clinical Procedures Safety. http://www.who.int/surgery/publications/ BestPracticeProtocolsCPSafety07.pdf (accessed 2011).

17. Youngleson $\mathrm{M}$, Nkurunziza $\mathrm{P}$, Jennings $\mathrm{K}$, et al. Improving a mother to child HIV transmission programme through health system redesign: quality improvement, protocol adjustment and resource addition. PLoS One 2010;5:e13891.

18. Maki J, Qualls M, White B, et al. Health impact assessment and shortterm medical missions: a methods study to evaluate quality of care. BMC Health Serv Res 2008;8:121.

19. Schwarz D. Implementing a hospital-based morbidity and mortality conference in remote rural Nepal. (in Preparation).

20. Donabedian A. Evaluating the Quality of Medical Care. The Milbank Memorial Fund Quarterly. New York, NY: Milbank Memorial Fund, Vol. 44, No. 3, Part 2, Health Services Research.

21. Nyaya Health. Nyaya Health Clinical Data Page. http://wiki. nyayahealth.org/ClinicData (accessed 2011).

22. Rabiee F. Focus-group interview and data analysis. Proc Nutr Soc 2004;63:655-60.

23. Kruk ME, Wladis A, Mbembati N, et al. Human resource and funding constraints for essential surgery in district hospitals in Africa: a retrospective cross-sectional survey. PLoS Med 2010;7:e1000242.

24. Nyaya Health. Nyaya Health Wiki Budget Page. 2011. http://wiki. nyayahealth.org/Budget (accessed 2011).
25. Nyaya Health. Nyaya Health Expansion Costing Sheet. 2011. http:// db.tt/Uuvqu5V (accessed 2011).

26. Marchal B, Dedzo M, Kegels G. Turning around an ailing district hospital: a realist evaluation of strategic changes at Ho Municipal Hospital (Ghana). BMC Public Health 2010;10:787.

27. Weiser TG, Haynes AB, Dziekan G, et al. Effect of a 19-item surgical safety checklist during urgent operations in a global patient population. Ann Surg 2010;251:976-80.

28. Duncan Maru. Following up: Update on Nyaya Health's Community Health Worker Program. http://blog.nyayahealth.org/2011/04/10/ chwfollowup (accessed 2011).

29. Nyaya Health. X-Ray Wiki Page. 2011. http://wiki.nyayahealth.org/XRay (accessed 2011)

30. Dindo D, Demartines N, Clavien PA. Classification of surgical complications: a new proposal with evaluation in a cohort of 6336 patients and results of a survey. Ann Surg 2004;240:205-13.

31. Procter LD, Davenport DL, Bernard AC, et al. General surgica operative duration is associated with increased risk-adjusted infectious complication rates and length of hospital stay. J Am Coll Surg 2010;210:60-5.e1.

32. U.S. Department of Health \& Human Services. Health Information Privacy. http://www.hhs.gov/ocr/privacy (accessed 2011).

33. Nyaya Health. Data Management Wiki Page. http://wiki.nyayahealth. org/DataManagement. 